\section{Understanding the origins of AIDS viruses}

SIR-Understanding the origins of the AIDS viruses is of obvious importance. Unfortunately, recent suggestions regarding the evolutionary history of HIV-1, and its relationship to the two other so-far elucidated groups of primate lentiviruses (HIV-2/SIV MAC $_{\text {and }}$ SIV $_{\mathrm{AGM}}$ ) are astonishingly divergent.

At one extreme, Fukasawa et al. suggest

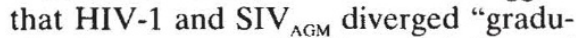
ally in concert with the evolution of primates", implying that the two viruses split millions of years ago. At the other, Smith et al. ${ }^{2}$ conclude that HIV-1 and HIV-2 may have evolved from a common ancestor "as recently as 40 years ago". The difference between these two dates is remarkable as HIV-1, HIV-2 and SIV $_{A G M}$ are approximately equidistantly related to one another (see below).

In fact, both dates seem unlikely in the light of present knowledge. First, nucleotide substitutions in retroviral (RNA) genomes accumulate about a million times faster than in the nuclear DNA of higher organisms $^{3.4}$. Because HIV-1 and SIV ${ }_{\text {AGM }}$ are only 20 times more divergent than human and Old World monkey DNA sequences ${ }^{5}$, it is likely that these two viruses have been separated for much less than 1,000 years. Second, serological evidence suggests ${ }^{6}$ that HIV-1 existed in central Africa about 30 years ago, before 1960 , and our recent analysis ${ }^{4}$ of diverse HIV-1 isolates suggests that their common ancestry goes back to about that time. As HIV-1 and HIV-2 are far more divergent than any two HIV-1 isolates ${ }^{2.7}$, it is very unlikely that HIV-1 and HIV-2 diverged only 40 years ago. We estimate that HIV- 1 , HIV-2 and SIV ${ }_{\mathrm{AGM}}$ all diverged about 150 years ago.

To study the evolutionary history of these rapidly evolving viruses, we examined the more conserved parts of the genome. The pol gene is best conserved, and phylogenetic analysis using nonprimate lentiviruses (such as visna) as outgroups reveals the likely relationships among the human and simian viruses (see figure). This phylogeny has two facets of immediate interest: the three main groups of primate lentiviruses, HIV-1, HIV-2 and SIV $_{\text {AGM }}$, diverged at about the same time (asterisk in the figure); and the branch lengths, indicating the degrees of divergence from that point, are similar down each descendant lineage, providing evidence for similar rates of molecular evolution among different groups. The latter result contradicts the suggestion ${ }^{8}$ that a more virulent virus, such as HIV-1, may evolve considerably more rapidly than less virulent ones, such as HIV-2 and SIV AGM $_{\text {. }}$

Because there seems to be a good molecular clock for HIV, it can be calibrated by placing only one time point on the phylogeny, to estimate how long ago the divergence at the asterisk took place. We have recently done this ${ }^{4}$ for the divergence times among HIV-1 isolates and obtained rates of evolution yielding divergence time estimates consistent with epidemiological data. HIV-1 ELI, isolated in 1983, was estimated to have diverged from the non-African isolates BRU, SF2 and RF around 1969. By extrapolation, the divergence of HIV-1, HIV-2 and SIV $_{\mathrm{AGM}}$ is estimated to have occurred between 140 and 160 years ago. Yokoyama et al. ${ }^{7}$ estimated that HIV-1 and HIV-2 diverged about 280 years ago, on the assumption that the lentivirus pol genes have evolved at the same rate as viral oncogenes, that is $0.5 \times 10^{-3}$ substitutions per (nonsynonymous) site per year ${ }^{3}$. Our rate estimate for the HIV-1 pol gene is higher $\left(0.96 \times 10^{-3}\right.$ in the conserved regions considered here), accounting for our shorter estimate of the divergence time.

Because HIV-1 and HIV-2 seem to have evolved at similar rates, we estimate that HIV-2 and SIV MAC $_{\text {diverged only }}$ about 30 years ago. Phylogenetic analysis? of a wider group of retroviruses supports the position of the root of the upper tree as indicated in the figure, and so suggests that the rate of evolution in the visna lineage has been similar to that in the
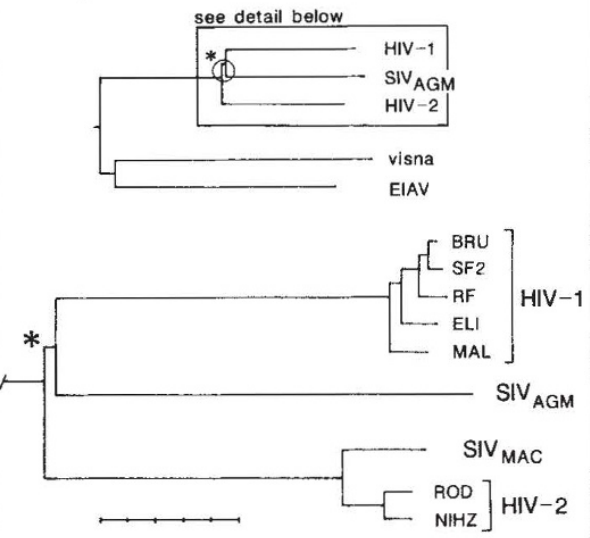

Phylogenetic relationships among lentiviruses. HIV, Human immunodeficiency virus; SIV, simian immunodeficiency virus (AGM, African green monkey; MAC, macaque); visna, ovine lentivirus; EIAV, equine infectious anaemia virus. Horizontal distances, proportional to the number of nucleotide substitutions along each branch; vertical separation for clarity only. Distances among viruses are in nonsynonymous nucleotide substitutions ${ }^{9}$ among pol genes (note that areas of the gene in which alignment is difficult were excluded, leaving 506 codons for comparison). Total length of scale bar, 0.05 substitutions per site. Phylogeny constructed using the neighbourjoining method ${ }^{10}$, which sequentially clusters sequences by minimizing the total branch length of the tree. The same branching order was obtained by the transformed distance method $^{11}$ using as references visna and EIAV. primate lentiviruses. Therefore, we can tentatively estimate that the latter separated from visna about 300 years ago.

Finally, note that both HIV-1 and HIV2 cause AIDS, whereas SIV ${ }_{\mathrm{AGM}}$ does not'. Our analysis suggests that all three viruses diverged at about the same time, suggesting that all three viruses were originally pathogenic, with SIV $_{\text {AGM }}$ having lost its pathogenicity after the divergence. Alternatively, if the three viruses were originally nonpathogenic, then HIV-1 and HIV2 must have acquired their pathogenicity independently after divergence.

Paul M. Sharp

Department of Genetics,

Trinity College, Dublin 2, Ireland

WEN-HSIUNG LI

Center for Demographic and Population Genetics,

University of Texas,

PO Box 20334,

Houston, Texas 77225 , USA

1. Fukasawa, M. et al. Nature 333, 457-461 (1988)

2. Smith, T.F., Srinivasan, A.. Schochetman, G., Marcus, M \& Myers, G. Nature 333, 573-575 (1988).

3. Gojobori, T. \& Yokoyama, S. Proc. natn. Acad. Sci U.S.A. 82, 4198-4202(1985)

4. Li, W-H., Tanimura, M. \& Sharp. P.M. Molec, biol. Evol. 5, 313-330 (1988).

5. Li, W-H., Tanimura, M. \& Sharp, P.M. J. molec. Evol. 25 , 330-342 (1987)

6. Nahmias, A.J. et al. Lancet i, 1279-1280 (1986)

7. Yokoyama, S., Chung, L. \& Gojobori, T. Molec. biol. Evol. 5, 237-251 (1988)

8. Penny. D. Nature 333, 494-495 (1988).

9. Li. W.-H., Wu, C.-I. \& Luo, C.-C. Molec. biol. Evol. 2 $150-174(1985)$

10. Saitou. N. \& Nei, M. Molec. biol. Evol. 4, 406-425 (1987).

11. Li, W.-H. Proc. natn. Acad. Sci. U.S.A. 78, 1085-1089 (1981).

\section{How to make models for behaviour of clouds}

In his commentary "Jumping the greenhouse gun", John Maddox states that "The well-known weakness of the climatic models, in this connection, is that real clouds (as distinct from average cloudiness) are on the face of it a source of negative feedback that may substantially moderate the expected size of the increase in temperature of the surface of the Earth." Here I explain why the sign of cloud feedback is very difficult to estimate by intuition and why, therefore, it is not surprising that recent general circulation model simulations with predicted (nonaverage) clouds give positive cloud feedbacks. To do this it is necessary to develop the following framework for the analysis of the feedbacks in the climatic system.

The net radiation at the top of the Earth's atmosphere can be expressed as $N$ $=N(\mathbf{E}, T, I)$, where $\mathbf{E}$ is a vector of quantities 'external' to the climate system (for example, the $\mathrm{CO}_{2}$ concentration), $T$ is the surface temperature, and $\mathbf{I}$ is a vector of quantities 'internal' to the climate system (for example, clouds). A small change in the energy flux, $\Delta N$, can be expressed as $\Delta N=\Delta Q-\left(G_{0}^{-1}-F\right) \Delta T$. Here $\Delta Q$ is the change in $N$ due to a 